\title{
'Tis the season. . .
}

\section{As the weather turns colder in the northern hemisphere, cases of colds and flu rise again, reminding us of the impact of pathogens on our lives.}

D espite the advent of antibiotics and vaccines, infectious diseases still account for around one-third of worldwide deaths, according to the World Health Organization. A molecular understanding of how pathogens cause disease is the object of intense research, and this issue of Nature Structural \& Molecular Biology features two examples of such efforts. On page 1214, Bernstein, Buchanan and colleagues provide insight into the mechanism of autotransporters, a specialized secretion pathway used by pathogenic Gram-negative bacteria to translocate virulence factors across their outer membranes. On page 1227, Garcia and colleagues present the structure of measles hemagglutinin, the glycoprotein responsible for viral binding to human cell receptors and a major target for neutralizing antibodies. The work extends our understanding of how this highly contagious virus attaches to and invades host cells, causing a disease that remains a major cause of mortality among young children, especially in developing areas.

Even in industrialized countries, pathogens still cast a long shadow. With the overuse of antibiotics and bacteria's striking ability to adapt and exchange genetic information, antibiotic-resistant strains have arisen and spread, posing a serious public health problem that is compounded by the paucity of new antibiotics. Methicillin-resistant Staphylococcus aureus, or MRSA (which also goes by the catchy moniker "superbug"), has been a leading cause of hospital-acquired infections. A recent surge of fatal cases of community-associated MRSA has frightened the public in the United States, and researchers have shown that these strains are even more virulent than their hospital cousins, secreting high levels of cytolysins that target neutrophils.

Though highly publicized in the media, severe cases of communityassociated MRSA are fortunately still rare. In contrast, acute upper respiratory viral infections such as colds and influenza (or flu) are highly prevalent, and the number of cases peaks during the cold season. According to the Centers for Disease Control and Prevention (CDC), up to $20 \%$ of the population in the United States gets the flu each year. The common cold is even more prevalent: the average adult catches $2-5$ colds per year, and the average child 7-10 (the latter number must seem like a gross underestimate to most parents). Usually colds are less severe than the flu, but both diseases can have more serious consequences for immunocompromised or elderly patients and young babies.

Several factors have been advanced to explain the higher incidence of colds and flu during the cold season: more time spent indoors, in crowded environments; low humidity causing the nasal mucosa to dry up; and even lower levels of vitamin D due to the lack of exposure to sunshine in the wintertime. Despite folklore, going outside underdressed or with your hair wet will not cause a cold. But don't discard the sweater and scarf just yet: a study at the Common Cold Centre at the University of Cardiff, UK, showed that volunteers who had their feet chilled in ice-cold water for 20 minutes

had a higher incidence of colds (29\%) in the next few days than controls who just put their feet in an empty basin (9\%), validating warnings from generations of concerned mothers. A possible explanation is that cold viruses may be carried asymptomatically, and exposure to cold may compromise immune defenses (for example, by causing vasoconstriction in the nasal mucosa), thus raising the rate of infection.

So how can one avoid coming down with the flu or a cold? A vaccine against the flu is available, and each year a new version, containing three inactivated influenza strains, is offered to the public, but its effectiveness will depend on the specific strain that infects you. There are no vaccines against the common cold, which can be caused by $\sim 200$ different kinds of viruses.

Both diseases are transmitted from person to person via droplets of fluids laden with viral particles released by a sick person. Transmission can also occur via fomites, the medical term for objects or surfaces that can transfer pathogens from one person to another, and the effectiveness of this route depends on the characteristics of each virus as well as on environmental conditions such as humidity and temperature. Thus, the simplest way to avoid catching an infection is to stay away from infected people, but that is usually beyond our control.

According to the CDC, the most effective measure to avoid the spread of infections is frequent hand washing, low-tech as it may seem. However, this simple habit might be losing popularity among the American general public, according to surveys conducted by the American Society of Microbiology and the Soap and Detergent Association. In 2007, 92\% of telephone survey respondents said they wash their hands after using a public restroom. However, monitoring of public facilities in four American cities showed the actual numbers were lower: only $76 \%$ of users did wash their hands, with a large gender difference ( $90 \%$ of women compared to $66 \%$ of men).

What can be done when one gets sick? Cutting down transmission is a considerate thing to do. People sick with colds or flu are most contagious during the initial phase of the infection. So if you are coming down with a cold or the flu, you should probably go home, bundle up with your latest issue of $N S M B$, get some rest and drink plenty of liquids. When sneezing or coughing, cover your mouth and nose, but not with your hand: a handkerchief or tissue is far preferable, and, in the absence of those, the inside of your arm is an acceptable shield.

And regardless of which side of the fluid droplet you stand on, wash your hands frequently. But do it properly: after applying soap, one should rub both hands vigorously for at least 20 seconds. The usual recommendation is to sing "Yankee Doodle", "The Alphabet Song" or the more universal "Happy Birthday" twice (and rather slowly). Then rinse and dry your hands.

'Tis the season. Let's spread the joy, not the germs. 